\title{
Abnormal Neural Synchrony in Schizophrenia
}

\author{
Kevin M. Spencer, Paul G. Nestor, Margaret A. Niznikiewicz, Dean F. Salisbury, Martha E. Shenton, and \\ Robert W. McCarley \\ Department of Psychiatry, Harvard Medical School/Veterans Affairs Boston Healthcare System, Brockton, Massachusetts 02301
}

\begin{abstract}
Schizophrenia has been conceptualized as a failure of cognitive integration, and abnormalities in neural circuitry (particularly inhibitory interneurons) have been proposed as a basis for this disorder. We used measures of phase locking and phase coherence in the scalprecorded electroencephalogram to examine the synchronization of neural circuits in schizophrenia. Compared with matched control subjects, schizophrenia patients demonstrated: (1) absence of the posterior component of the early visual gamma band response to Gestalt stimuli; (2) abnormalities in the topography, latency, and frequency of the anterior component of this response; (3) delayed onset of phase coherence changes; and (4) the pattern of anterior-posterior coherence increases in response to Gestalt stimuli found in controls was replaced by a pattern of interhemispheric coherence decreases in patients. These findings support the hypothesis that schizophrenia is associated with impaired neural circuitry demonstrated as a failure of gamma band synchronization, especially in the $40 \mathrm{~Hz}$ range.
\end{abstract}

Key words: schizophrenia; gamma band EEG; phase synchrony; wavelet analysis; visual perception; inhibitory interneurons

\section{Introduction}

As early as 1911, Bleuler had described schizophrenia as a "splitting of the psychic functions” (Bleuler, 1950/1911), in which various aspects of thought and personality were disintegrated. In the modern era, it has been proposed that the cognitive and affective impairments associated with schizophrenia may be related to a failure to integrate the activity of local and distributed neural circuits (Friston and Frith, 1995; Grunze et al., 1996; Selemon and Goldman-Rakic, 1999; Benes, 2000; Lewis and GonzalezBurgos, 2000; Whittington et al., 2000). This hypothesis has been supported by postmortem studies demonstrating abnormalities in the morphology and distribution of certain cortical neurons in schizophrenia (Selemon and Goldman-Rakic, 1999; Benes, 2000; Lewis and Gonzalez-Burgos, 2000; Whittington et al., 2000), particularly inhibitory interneurons. Evidence suggests that interactions between inhibitory interneurons (subject to NMDA modulation from excitatory pyramidal cells) are an important substrate of synchronous neural activity in the upper beta and gamma bands (20-100 Hz) (Grunze et al., 1996; Whittington et al., 2000; McBain and Fisahn, 2001), which may play an important role in the formation and selection of neural representations (Engel et al., 2001; Varela et al., 2001). Hence, the study of highfrequency synchrony in the scalp-recorded electroencephalogram (EEG) may shed light on the functional integrity of neural circuits in schizophrenia and on the relationship between neural synchrony and thought processes in general.

Phase locking is a highly sensitive measure of neural synchronization in the EEG (Tallon-Baudry and Bertrand, 1999). Spec-

Received March 10, 2003; revised June 23, 2003; accepted June 24, 2003.

This study was supported by National Institute of Mental Health Grant 40799 to R.W.M., Fellowship 13022 to K.M.S., and Training Grant 016259. The Wavelet software was provided by C. Torrence and G. Compo (available at http://paos.colorado.edu/research/wavelets/).

Correspondence should be addressed to Dr. Robert W. McCarley, Harvard Medical School/Veterans Affairs Boston Healthcare System, Psychiatry 116A, 940 Belmont Street, Brockton, MA 02301. E-mail: robert_mccarley@hms.harvard.edu.

Copyright $\odot 2003$ Society for Neuroscience $\quad$ 0270-6474/03/237407-05\$15.00/0 tral power has been commonly used as a putative measure of neural synchrony in the scalp-recorded EEG, but, in fact, this measure is sensitive only to the amplitude of oscillations and not the phase, so it cannot directly measure temporal correlations of oscillatory activity. In contrast, phase locking measures the variance of EEG phase across single trials independently of amplitude, making it sensitive to small amplitude oscillations that would not be detected with power measures. Furthermore, phase locking can be computed between electrode sites (here termed "phase coherence"), providing a measure of temporally synchronous interactions in distributed neural networks (Varela et al., 2001), which may also be abnormal in schizophrenia.

We used phase locking and phase coherence to examine whether high-frequency neural synchronization associated with the perception of visual Gestalts is abnormal in schizophrenia patients. Previous studies of healthy individuals have reported enhancements of gamma band power (Tallon-Baudry and Bertrand, 1999; Herrmann et al., 1999) and phase locking (Rodriguez et al., 1999) when coherent objects are perceived. In this study, schizophrenic (SZ) and matched normal control (NC) subjects discriminated between illusory square and control stimuli (Square/No-Square conditions) (Fig. 1A). Several abnormalities were found in the schizophrenia patients' phase locking and phase coherence responses, particularly to illusory square stimuli, which presumably engage synchronization mechanisms that support visual feature binding. These results provide further evidence for the hypothesis that neural synchronization is abnormal in schizophrenia.

\section{Materials and Methods}

Participants. Inclusion criteria for patients and controls included: (1) age between 18 and 50 years; (2) right handed; (3) no history of electroconvulsive treatment; (4) no history of neurological illness; (5) no history of alcohol or drug dependence, or abuse within the last year, or long duration ( $>1$ year) of past abuse; (6) no present medication for medical disorders that would have deleterious EEG, neurological, and/or cognitive functioning consequences; (7) verbal intelligence quotient above 75; 


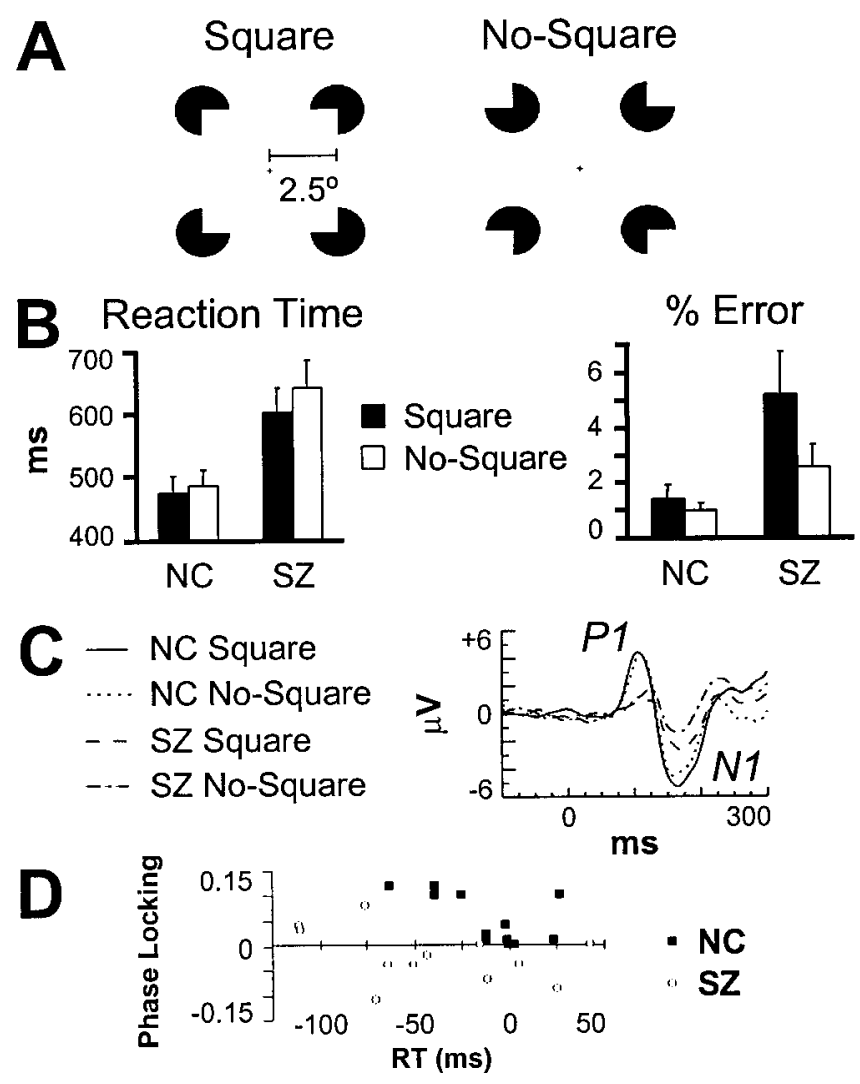

Figure 1. Illusory square discrimination task. $A$, Stimuli. The ratio of inducer radius to length of square was 0.2. B, Behavioral performance data (bars indicate SE). C, VEPs at electrode site P06. D, Relationship between the Square minus No-Square RT ( $x$-axis) and occipital phaselocking ( $y$-axis) effects. The greater the RT savings, the larger the phase-locking effects for NC but not SZ.

(8) no alcohol use in the $24 \mathrm{hr}$ before testing; and (9) an ability and desire to cooperate with our experimental procedures as demonstrated by giving informed consent (following Harvard Medical School and Veterans Affairs Boston Healthcare System guidelines)

Fourteen schizophrenia patients [diagnosed according to the Diagnostic and Statistical Manual of Mental Disorders, fourth edition (American Psychiatric Association, 1994)] participated in the study. Two patients' data were unusable, one because of artifacts, the other because of an excessive error rate $(\sim 50 \%)$. The final sample for the SZ group consisted of 12 chronic medicated male patients (mean \pm SD; age, $45 \pm 9.2$ years; illness duration, $21 \pm 5.1$ years). The NC group consisted of 12 healthy males (age, $47 \pm 5.7$ years) matched with the SZ group on the basis of age $\left(F_{(1,22)}=0.311\right.$; NS) and parental socioeconomic status. Five patients were receiving conventional neuroleptics, six patients received atypical antipsychotics, and one patient received both types. Mean equivalent chlorpromazine dosage was $519 \pm 539 \mathrm{mg}$.

Stimuli and experimental procedures. Subjects fixated a central cross and responded with a button press according to whether an illusory square (Fig. $1 A$ ) was present or absent (response hands counterbalanced across subjects). Stimuli remained on the monitor until $300 \mathrm{msec}$ after a response had been made. If no response had been made by $2000 \mathrm{msec}$ after stimulus onset, the trial was ended, and the next trial began (1000 msec intertrial interval). Subjects performed a practice block and two experimental blocks, each consisting of 45 trials per condition.

Electrophysiological recording and processing. The EEG was recorded (0.01-100 Hz; $500 \mathrm{~Hz}$ digitization) with tin electrodes at 16 scalp sites (F3/Fz/F4, C3/Cz/C4, P3/Pz/P4, O1/Oz/O2, P7/P8, PO5/PO6) and the right mastoid, referenced to the left mastoid. The vertical electrooculogram (EOG) was recorded at Fp1, and the horizontal EOG was recorded at the outer canthi of the eyes. Electrode impedances were $<5$ $\mathrm{k} \Omega$. Error and no-response trials were excluded from analyses. Independent component analysis (Makeig et al., 1996) was used to identify eyeblink artifacts by their distinct topography and to remove their contribution to each subject's data. Corrected single-trial epochs were rereferenced to averaged mastoids. For the SZ and NC groups, there were averages of 85.5 and 87.5 trials per condition, respectively.

Data analysis. The complex Morlet wavelet transform (six-cycle length) (Torrence and Compo, 1998) was applied to the $20-60 \mathrm{~Hz}$ frequency range of the EEG in the -200 to $+300 \mathrm{msec}$ period relative to stimulus onset. Wavelet frequencies were centered at 20.3, 22.1, 24.1, 26.3, 28.7, 31.2, 34.1, 37.2, 40.5, 44.2, 48.2, 52.6, and 57.3 Hz. Phase locking was computed as one minus the circular variance of phases at each time point, wavelet frequency, and electrode (Tallon-Baudry and Bertrand, 1999). Phase coherence was computed as one minus the circular variance of the phase differences between pairs of electrodes at each time point and wavelet frequency (Lachaux et al., 1999). Every unique pair of the 16 scalp electrodes (120 pairs) was used in this analysis. Prestimulus baseline levels $(-200$ to $-50 \mathrm{msec})$ were subtracted from each time-frequency map.

The occipital component of the visual evoked gamma band response was measured as the average phase-locking value within the $80-120 \mathrm{msec}$ and $31-44 \mathrm{~Hz}$ ranges. Analysis of the fronto-central component was based on peak values at midline and right hemisphere sites where it was maximal, with latency and frequency ranges selected to avoid overlap with the visual-evoked potentials (VEPs). The latency range was 80-160 msec for both groups and conditions, except for NC subjects in the Square condition, in which it was $80-110 \mathrm{msec}$. Frequency ranges for both groups were $34-58$ (Square) and $28-58 \mathrm{~Hz}$ (No-Square).

For phase coherence analyses of the Square and No-Square conditions, values were compared with the mean prestimulus baseline in two-tailed paired $t$ tests at each time-frequency element $(0-300 \mathrm{msec}$ period) for each group. In the Square versus No-Square comparison, $t$ tests were computed between values for the respective conditions. Significance values were corrected for the number of frequency bins $(n=4$; Fig. 3$)$, resulting in a statistical criterion of $p=0.0125(t= \pm 2.98)$. A temporal threshold was applied to the statistical maps such that any period of coherence change above the $t$ threshold had to be at least one cycle in duration at that particular frequency. Coherence changes were summed within each frequency bin across four time bins (Fig. 4).

VEP amplitudes were measured at sites $\mathrm{O} 1 / \mathrm{O} 2, \mathrm{P} 7 / \mathrm{P} 8$, and PO5/PO6. Latency ranges for the $\mathrm{P} 1$ were $76-126 \mathrm{msec}(\mathrm{NC})$ and $78-138 \mathrm{msec}$ (SZ), and for the N1, 126-212 msec (NC) and 138-212 msec (SZ).

Phase locking and VEP amplitude were statistically analyzed using ANOVA with the design group (SZ/NC) X stimulus (Square/No-Square) $\mathrm{X}$ site (relevant electrode sites). Effects on group level coherence changes were analyzed using the $\chi^{2}$ goodness-of-fit test, comparing the distributions of coherence changes across frequency bins.

\section{Results}

\section{Behavioral performance}

$\mathrm{NC}$ and SZ subjects had similar median reaction time (RT) and error rate patterns (Fig. $1 B$ ). In general, subjects made more errors $\left(F_{(1,22)}=4.99 ; p<0.05\right)$ and had shorter RTs $\left(F_{(1,22)}=\right.$ 9.92; $p<0.01)$ on Square than No-Square trials. This shorter RT for Squares also was present within the SZ group $\left(F_{(1,11)}=7.61\right.$; $p<0.05)$ but not within the NC group $\left(F_{(1,11)}=2.32\right.$; NS). Whereas the SZ patients' low overall error rate (3.9\%) indicated that they did not have any particular difficulties with the task, they did make more errors $\left(F_{(1,22)}=5.97 ; p<0.05\right)$ and had longer RTs $\left(F_{(1,22)}=9.06 ; p<0.01\right)$ than the NC group.

\section{VEPs}

The most notable group difference in the VEPs (Fig. 1C) was that the P1 component was larger in amplitude for NC than SZ subjects across stimulus conditions $\left(F_{(1,22)}=6.22 ; p<0.05\right)$. There was a trend for $\mathrm{N} 1$ component amplitude to be larger in the NC than the SZ group $\left(F_{(1,22)}=3.11 ; p=0.09\right)$, and across all sub- 

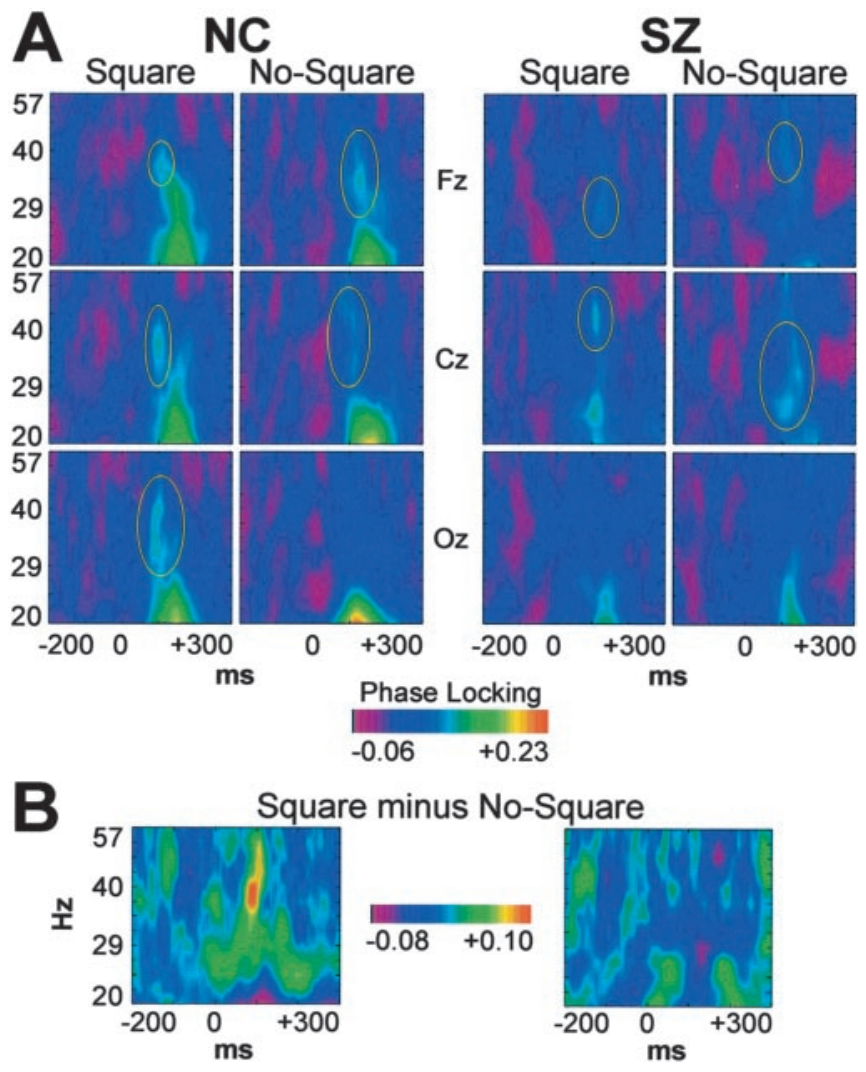

Figure 2. Group average time-frequency maps of phase locking. $A$, Midline frontal (Fz), central ( $\mathrm{z}$ ), and occipital ( $\mathrm{Oz}$ ) sites. The early evoked gamma band response (circled in yellow) can be distinguished from the low-frequency VEP. B, Square minus No-Square difference map at the left occipital site (01).

jects, the N1 was significantly larger for Square than No-Square $\operatorname{stimuli}\left(F_{(1,22)}=8.66 ; p<0.01\right)$.

\section{Phase-locking data}

For both the NC and SZ groups, visual stimuli evoked an early phase-locking response in the $24-48 \mathrm{~Hz}$ range that began as early as $80 \mathrm{msec}$ and did not overlap with the low-frequency VEP at frontal, central, and occipital sites (Fig. 2A). Previous studies have differentiated this early evoked gamma band response into anterior and posterior components (Tallon-Baudry et al., 1997), and in this study, different effects on the response were indeed found at occipital and fronto-central sites.

The most striking difference between the SZ and NC groups was in the occipital component of the response (group X stimulus; $\left.F_{(1,22)}=7.29 ; p<0.05\right)$. In the NC group data, Square stimuli evoked this response maximally at the left occipital site, but there was no response to the No-Square stimuli $\left(F_{(1,11)}=10.2 ; p<\right.$ $0.01)$. For the $S Z$ subjects, however, neither stimulus evoked the response $\left(F_{(1,11)}=0.515\right.$; NS). These group differences are highlighted in Figure $2 \mathrm{~B}$. To try to determine the functional significance of the occipital phase-locking response, we computed nonparametric Spearman's $\rho$ correlation coefficients between the Square minus No-Square differences in phase synchrony and RT across subjects in the NC group. This correlation was negative and statistically significant ( $\rho=-0.677 ; p<0.05$; two-tailed), indicating that the larger the response, the larger the RT advantage for Square compared with No-Square stimuli (Fig. 1D). No such correlation was found for the SZ group. These data suggest that the occipital phase-locking response may reflect a feature-binding mechanism in visual cortex that underlies more efficient task performance for healthy individuals, but not persons with schizophrenia.

The fronto-central component of the early evoked gamma band response was abnormal in topography, latency, and frequency for the SZ group. Whereas for both groups this response was larger for No-Square than Square stimuli (NC: $F_{(1,11)}=5.70$; $\left.p<0.05 ; \mathrm{SZ}: F_{(1,11)}=5.19 ; p<0.05\right)$, it was larger at central than frontal electrode sites for SZ (group X site; $F_{(1,22)}=10.0 ; p<$ $\left.0.01 ; \mathrm{SZ}: F_{(1,11)}=24.5 ; p<0.001\right)$ but not NC subjects $\left(F_{(1,11)}=\right.$ 0.00695 ; NS). The latency of the fronto-central response to Square stimuli was longer for SZ than NC subjects (116.9 vs 99.2 msec; group X stimulus; $F_{(1,22)}=5.66 ; p<0.05$; group: $F_{(1,22)}=$ $24.1 ; p<0.0001)$, but there was no difference for No-Square stimuli ( 117.3 vs $119.1 \mathrm{msec} ; F_{(1,22)}=0.046$; NS). Last, the frequency of the response to No-Square stimuli was lower for SZ than NC subjects at central sites (35.3 vs $42.4 \mathrm{~Hz}$; group X stimulus $X$ site; $F_{(1,22)}=5.08 ; p<0.05$; group: $F_{(1,22)}=4.45 ; p<$ 0.05 ), but there was no difference for Square stimuli ( 44.4 vs 41.5 $\left.\mathrm{Hz} ; F_{(1,22)}=1.04 ; \mathrm{NS}\right)$.

In summary, the early evoked gamma band response differed between the SZ and NC groups in several ways, being absent for the occipital component and abnormal in topography, latency, and frequency for the fronto-central component. These results support the hypothesis that gamma band synchronization is abnormal in schizophrenia. The different patterns observed for the VEPs and phase-locking responses indicate that the latter are not artifacts of the low-frequency VEPs.

To test for potential confounds with medication, we computed Spearman's $\rho$ correlations between antipsychotic medication dosage in chlorpromazine equivalents and the four effects listed above. None of the correlations approached significance. Next, we analyzed whether any of the above effects were related to error rate, age, illness duration, or SZ symptoms as measured by the Positive and Negative Symptom Scale (PANSS) (Kay et al., 1987). Whereas there were no correlations for errors, age, or illness duration, the frequency of the No-Square response at central sites correlated negatively with major categories of the PANSS: total symptoms $(-0.72 ; p<0.01)$, positive symptoms $(-0.58 ; p<0.05)$, negative symptoms $(-0.62 ; p<0.05)$, and general symptoms $(-0.68 ; p<0.05)$. Particularly notable in view of our hypothesis about neural synchrony are the correlations with specific items, such as delusions $(-0.68 ; p<0.05)$, conceptual disorganization $(-0.66 ; p<0.05)$, and poor attention $(-0.83$; $p<$ 0.001 ). Thus, the lower the frequency of the early evoked gamma band response, the greater the degree of SZ symptoms.

\section{Phase coherence data}

Next, we examined phase coherence, which is indicative of coupling between distant brain regions (Varela et al., 2001). Periods of coherence change relative to the baseline (coherence increases and decreases) were computed between each pair of electrodes for each group using a statistical parametric mapping method (see Materials and Methods). The number of coherence changes for each group and condition are presented in Figure 3. It can be seen that the NC group had far more coherence increases than the SZ group, particularly in the $20-26 \mathrm{~Hz}$ bin. Inspection of the coherence time-frequency maps revealed that this effect was because of the presence of the VEP in this frequency range, so subsequent analyses focused on the higher frequency bins.

Three main differences between the groups were observed (Fig. 3). First, the NC group had more coherence increases than the SZ group overall (249 vs 91) and in both the Square $\left(\chi_{(1)}^{2}=\right.$ 89.5; $p<0.0001)$ and the No-Square $\left(\chi_{(1)}^{2}=8.99 ; p<0.01\right)$ conditions. Second, the NC and SZ groups had different fre- 


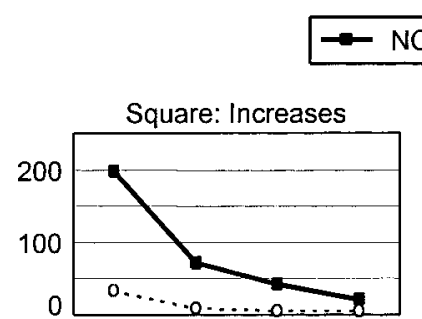

\section{$\cdot \infty \cdot S Z$}
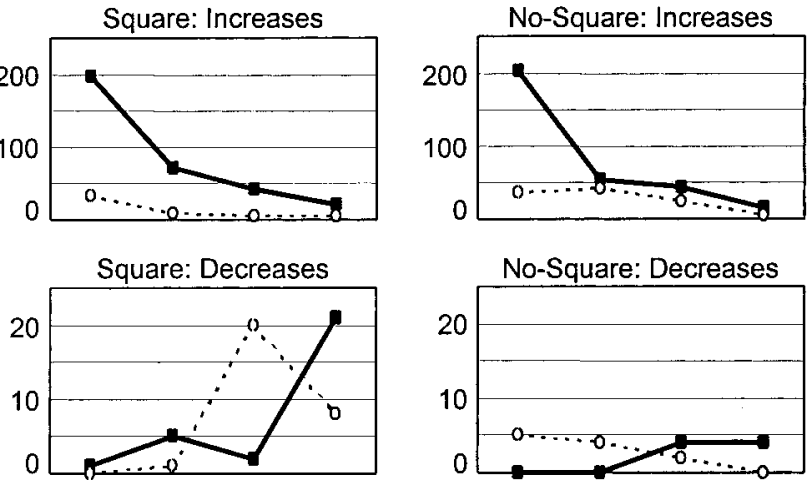

Square - No-Square
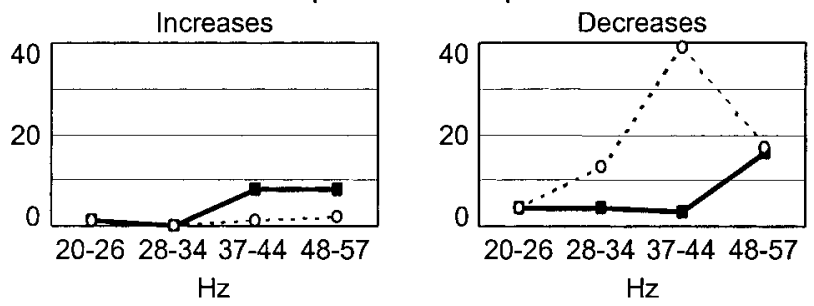

Figure 3. Phase coherence results. The distributions of the number of coherence changes (increases and decreases) are plotted across frequency bins in each stimulus condition and the Square minus No-Square comparison. Note the different $y$-axis scales for each row of plots.

quency band distributions of coherence decreases in the Square condition $\left(\chi_{(2)}^{2}=113 ; p<0.0001\right)$; the SZ distribution peaked at $37-44 \mathrm{~Hz}$, and the NC distribution peaked at $48-57 \mathrm{~Hz}$. Third, in the Square minus No-Square comparison, coherence changes were mainly decreases in coherence for the SZ group. The frequency band distribution of coherence decreases differed between groups $\left(\chi_{(2)}^{2}=\right.$ $115 ; p<0.0001)$ : $S Z$ coherence decreases showed a peak at $37-44 \mathrm{~Hz}$ $\left(\chi_{(2)}^{2}=17.0, p<0.001\right)$, where there were significantly more coherence decreases than the NC group $\left(\chi_{(1)}^{2}=30.9 ; p<0.0001\right)$, especially in the Square condition (Fig. 3). In summary, the phase coherence data point to abnormalities of coherence in longdistance neural synchronization in schizophrenia.

The spatio-temporal distributions of coherence changes in the $37-44 \mathrm{~Hz}$ bin (Fig. 4) revealed several patterns. For the NC group, coherence changes began in the $0-75 \mathrm{msec}$ interval and were concentrated in the 75-150 msec interval, coinciding with the early evoked gamma band response, and showed a roughly similar pattern of anterior-posterior coherence increases in the Square and No-Square conditions. Consequently, there were few differences in coherence in the Square minus No-Square comparison. For the SZ group, the onset of coherence changes was delayed, not beginning until after $75 \mathrm{msec}$. In the Square condition, the number of coherence changes (mainly decreases) was largest in the 225-300 msec interval and involved mostly interhemispheric interactions between posterior sites. In contrast, in the No-Square condition, the coherence changes were predominantly anterior-posterior increases in the 75-150 and 150-225 msec intervals, more in agreement in latency and directionality with those in the NC data. In the Square minus No-Square comparison, the SZ subjects displayed a consistent pattern of interhemispheric coherence decreases between posterior sites across the $0-300$ msec period, in contrast to the NC group. The electrodes most often

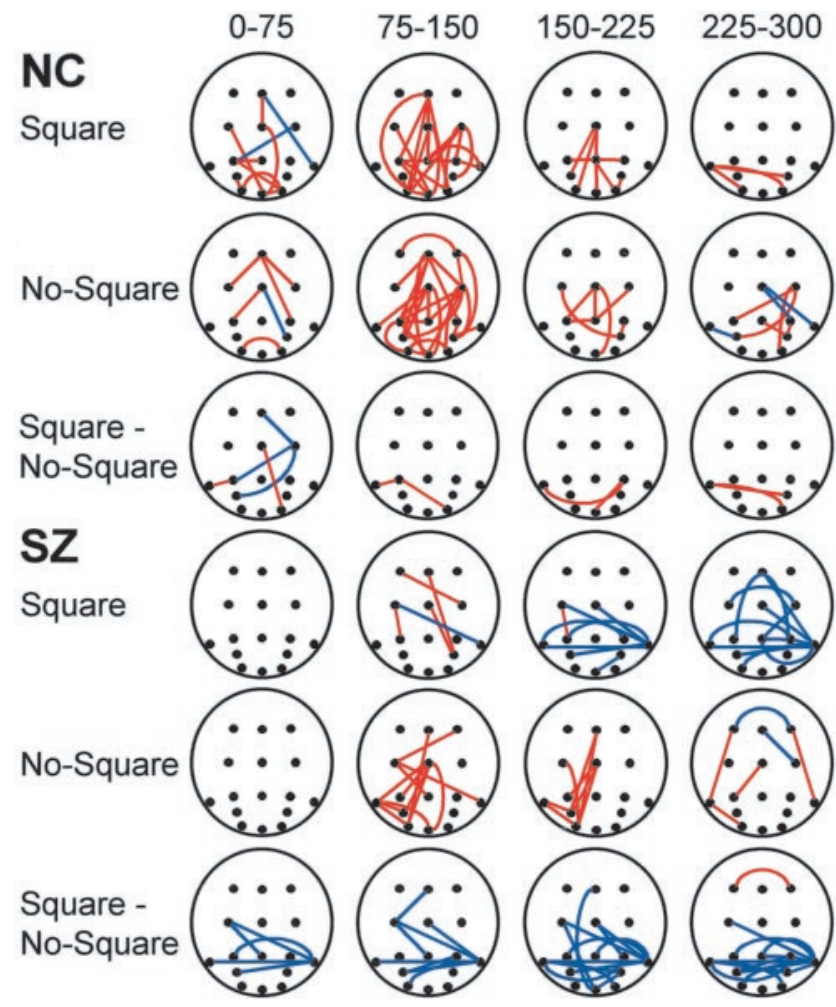

Figure 4. Phase coherence changes in the $37-44 \mathrm{~Hz}$ frequency bin plotted in space and time. Circles indicate electrode positions, with frontal sites at top and occipital sites at bottom. Red lines mark coherence increases between electrode sites, and blue lines mark coherence decreases.

involved in these coherence decreases were $\mathrm{P} 7$ and $\mathrm{P} 8$, which lie over posterior temporal visual areas. Thus, phase coherence changes generally had a delayed onset for SZ compared with NC subjects and differed maximally between the groups in the Square condition, in which the SZ pattern consisted of coherence decreases between posterior sites, contrasting with the $\mathrm{NC}$ pattern of coherence increases along the anterior-posterior axis.

These results demonstrate that long-range neural synchronization in the upper beta and gamma bands of the EEG is impaired in schizophrenia, particularly in the $40 \mathrm{~Hz}$ range. As in the phaselocking data, the illusory square stimuli were especially associated with a lack of coherence. It should be noted that while possible effects of volume conduction cannot be ruled out from these data, the calculation of phase coherence relative to the prestimulus baseline reduces such a confound by eliminating any overall level of coherence. Also, volume conduction would be expected to bias the spatial distribution of coherence patterns to adjacent electrodes, but this pattern was not observed (cf. Rodriguez et al., 1999).

\section{Discussion}

We found that schizophrenia was associated with abnormal phaselocking and phase coherence responses to visual stimuli in a Gestalt perception task, supporting the hypothesis that abnormal neural circuit function may be an underlying cause of schizophrenia. The patients' phase-locking and coherence patterns were particularly abnormal in the Square condition, in which the perception of the illusory object presumably engages feature-binding mechanisms that rely on neural synchrony (Engel et al., 2001). The correlation between the RT and phase-locking effects for the NC subjects suggests that they performed the task using an early perceptual mechanism involving temporal coding in occipital cortex. The ability of the SZ 
patients to perform the Gestalt detection task, albeit less efficiently than the NC subjects, could be the result of a phase-locking effect that was not detectable in the scalp EEG, or the patients might have performed the task using some later stage of analysis.

Earlier studies have documented SZ abnormalities in measures of gamma band power or amplitude in the EEG (Kwon et al., 1999; Haig et al., 2000; Kissler et al., 2000; Gordon et al., 2001; Green et al., 2003). Also, in our laboratory, we previously examined stimulus-driven phase locking in the EEG to steady-state auditory stimulation. Abnormal phase locking to $40 \mathrm{~Hz}$ click trains was found in the average steady-state auditory-evoked responses of schizophrenia patients (Kwon et al., 1999). Compared with matched healthy controls, the patients' steady-state responses were slower to phase lock with the stimulus trains and never reached the same degree of maximal phase locking. Those findings were consistent with the hypothesis that neural synchrony is abnormal in schizophrenia. The results of the present study confirm and extend these observations using a method with greater sensitivity to neural synchronization and with a task that engages feature-binding processes. We note that the power of the early gamma band response did not differ between conditions or subject groups, which highlights the sensitivity of the phase-locking method.

Several recent studies have demonstrated that a critical neural substrate of high-frequency oscillatory activity in the cortex lies in interactions between inhibitory interneurons, mediated both by $\mathrm{GABA}_{\mathrm{A}}$ synapses and gap junctions (Whittington et al., 2000; McBain and Fisahn, 2001). Because abnormalities in inhibitory interneurons have been documented in postmortem studies of SZ brains, synchronous neural activity could be disturbed in schizophrenia through the dysfunction of inhibitory interneuron networks, as well as through impaired connections from interneurons to pyramidal cells (Volk et al., 2002). Also, given the evidence for NMDA receptor dysregulation in schizophrenia (Javitt and Zukin, 1991; Tsai and Coyle, 2002), another possibility is that abnormalities in pyramidal cells (Lewis and GonzalezBurgos, 2000) could impair the NMDA-mediated glutamate projections from pyramidal cells to interneurons, which play a key role in regulating interneuron activity (Grunze et al., 1996).

The phase coherence data are consistent with a growing body of functional (Fletcher et al., 1999; Meyer-Lindenberg et al., 2001) and anatomical (Kubicki et al., 2002) studies pointing to impairments in the connectivity of distributed cortical networks in schizophrenia. The present data demonstrate that functional connectivity between brain regions is abnormal at the millisecond time scale in schizophrenia and suggest that interhemispheric interactions may be particularly affected. In conclusion, we suggest that in schizophrenia, the synchronization of gamma band neural activity is abnormal, and this abnormality is implicated in some of the symptoms and signs of this disorder. These data also suggest that neural synchrony in the $40 \mathrm{~Hz}$ range of the EEG may be important in normal brain function.

\section{References}

Benes FM (2000) Emerging principles of altered neural circuitry in schizophrenia. Brain Res Rev 31:251-269.

Bleuler E (1950/1911) Dementia praecox or the group of schizophrenias. New York: International UP.

American Psychiatric Association (1994) Diagnostic and statistical manual of mental disorders, Ed 4. Washington, DC: American Psychiatric Association.

Engel AK, Fries P, Singer W (2001) Dynamic predictions: oscillations and synchrony in top-down processing. Nat Rev Neurosci 2:704-716.
Fletcher P, McKenna PJ, Friston KJ, Frith CD, Dolan RJ (1999) Abnormal cingulate modulation of fronto-temporal connectivity in schizophrenia. NeuroImage 9:337-342.

Friston KJ, Frith CD (1995) Schizophrenia: a disconnection syndrome? Clin Neurosci 3:89-97.

Gordon E, Williams L, Haig AR, Wright J, Meares RA (2001) Symptom profile and "gamma" processing in schizophrenia. Cogn Neuropsychiatry 6:7-19.

Green MF, Mintz J, Salveson D, Nuechterlein KH, Breitmeyer B, Light GA, Braff DL (2003) Visual masking as a probe for abnormal gamma range activity in schizophrenia. Biol Psychiatry 53:1113-1119.

Grunze HC, Rainnie DG, Hasselmo ME, Barkai E, Hearn EF, McCarley RW, Greene RW (1996) NMDA-dependent modulation of CA1 local circuit inhibition. J Neurosci 16:2034-2043.

Haig AR, Gordon E, De Pascalis V, Meares RA, Bahramali H, Harris A (2000) Gamma activity in schizophrenia: evidence of impaired network binding? Clin Neurophysiol 111:1461-1468.

Herrmann CS, Mecklinger A, Pfiefer E (1999) Gamma responses and ERPs in a visual classification task. Clin Neurophysiol 110:636-642.

Javitt DC, Zukin SR (1991) Recent advances in the phencyclidine model of schizophrenia. Am J Psychiatry 48:1301-1308.

Kay SR, Fiszbein A, Opler LA (1987) The positive and negative syndrome scale (PANSS) for schizophrenia. Schizophr Bull 13:261-276.

Kissler J, Muller MM, Fehr T, Rockstroh B, Elbert T (2000) MEG gamma band activity in schizophrenia patients and healthy subjects in a mental arithmetic task and at rest. Clin Neurophysiol 111:2079-2087.

Kubicki M, Westin C-F, Maier SE, Frumin M, Nestor PG, Salisbury DF, Kikinis R, Jolesz FA, McCarley RW, Shenton ME (2002) Uncinate fasciculus findings in schizophrenia: a magnetic resonance diffusion tensor imaging study. Am J Psychiatry 159:813-820.

Kwon JS, O’Donnell BF, Wallenstein GV, Greene RW, Hirayasu Y, Nestor PG, Hasselmo ME, Potts GF, Shenton ME, McCarley RW (1999) Gamma frequency-range abnormalities to auditory stimulation in schizophrenia. Arch Gen Psychiatry 56:1001-1005.

Lachaux J-P, Rodriguez E, Martinerie J, Varela FJ (1999) Measuring phase synchrony in brain signals. Hum Brain Mapp 8:194-208.

Lewis DA, Gonzalez-Burgos G (2000) Intrinsic excitatory connections in the prefrontal cortex and the pathophysiology of schizophrenia. Brain Res Bull 52:309-317.

Makeig S, Bell AJ, Jung T-P, Sejnowski TJ (1996) Independent component analysis of electroencephalographic data. Adv Neural Inf Process Syst 8:145-151.

McBain CJ, Fisahn A (2001) Interneurons unbound. Nat Rev Neurosci 2:11-23.

Meyer-Lindenberg A, Poline J-B, Kohn PD, Holt JS, Egan MF, Weinberger DR, Berman KF (2001) Evidence for abnormal cortical functional connectivity during working memory in schizophrenia. Am J Psychiatry 158:1809-1817.

Rodriguez E, George N, Lachaux J-P, Martinerie J, Renault B, Varela FJ (1999) Perception's shadow: long-distance synchronization of human brain activity. Nature 397:430-433.

Selemon LD, Goldman-Rakic PS (1999) The reduced neuropil hypothesis: a circuit based model of schizophrenia. Biol Psychiatry 45:17-25.

Tallon-Baudry C, Bertrand O (1999) Oscillatory gamma activity in humans and its role in object representation. Trends Cogn Sci 3:151-162.

Tallon-Baudry C, Bertrand O, Wienbruch C, Ross B, Pantev C (1997) Combined EEG and MEG recordings of visual $40 \mathrm{~Hz}$ responses to illusory triangles in man. NeuroReport 8:1103-1107.

Torrence C, Compo GP (1998) A practical guide to wavelet analysis. Bull Am Meteor Soc 79:61-78.

Tsai G, Coyle JT (2002) Glutamatergic mechanisms in schizophrenia. Annu Rev Pharmacol Toxicol 42:165-179.

Varela FJ, Lachaux J-P, Rodriguez E, Martinerie J (2001) The brainweb: phase synchronization and large-scale integration. Nat Rev Neurosci 2:229-239.

Volk DW, Pierri JN, Fritschy J-M, Auh S, Sampson AR, Lewis DA (2002) Reciprocal alterations in pre- and postsynaptic inhibitory markers at chandelier cell inputs to pyramidal neurons in schizophrenia. Cereb Cortex 12:1063-1070.

Whittington M, Faulkner HJ, Doheny HC, Traub RD (2000) Neuronal fast oscillations as a target site for psychoactive drugs. Pharmacol Ther 86 : 171-190. 